\title{
Certificación laboral y empleabilidad de los beneficiarios del programa Impulsa Perú, 2011-20161
}

\section{Labor certification and employability of the Impulsa Peru program beneficiaries, 2011-2016}

\author{
Christian Omar Silva Suárez ${ }^{2}$ \\ Ministerio de Trabajo y Promoción del Empleo \\ cislvas@trabajo.gob.pe \\ https://orcid.org/0000-0002-1772-1395
}

Aceptado: 01/08/2019

\section{RESUMEN}

El estudio se centró en los resultados obtenidos a través del servicio de Certificación de Competencias Laborales brindado por el Programa Impulsa Perú del Ministerio de Trabajo y Promoción del Empleo (MTPE) durante los años 2011 - 2016, de acuerdo a la normativa establecida por la Dirección General de Formación Profesional y Capacitación Laboral (DGFPCL), así como a los lineamientos del Programa Presupuestal 0116 "Mejoramiento de la Empleabilidad e Inserción Laboral PROEMPLEO". Estos resultados se midieron en base a dos indicadores clave como son el nivel de experiencia laboral y el nivel de salario, los cuales están ligados a la empleabilidad de una persona. Para ello, se tomaron cuatro muestras cuyo tamaño por muestra fue de 1,804 beneficiarios con competencias laborales certificadas y se compararon con los 1,804 beneficiarios no competentes. Se pudo concluir que la permanencia en el mercado laboral tuvo significancia en el análisis de varianza y en dos de las cuatro muestras, mostrando diferencias de 1 a 3 meses. Por otro lado,

(C) Los autores. Este artículo es publicado por Pensamiento Crítico de la Facultad de Ciencias Económicas, Universidad Nacional Mayor de San Marcos. Este es un artículo de acceso abierto, distribuido bajo los términos de la licencia Creative Commons Atribucion - No Comercia_Compartir Igual 4.0 Internacional. (http:// creativecommons.org/licenses/by-nc-sa/4.0/) que permite el uso no comercial, distribución y reproducción en cualquier medio, siempre que la obra original sea debidamente citada. 
el salario mostró significancia tanto en el análisis de varianza como para las cuatro muestras comparadas, con diferencias entre S/ 30 a S/ 70 soles.

Palabras clave: Certificación de competencias laborales; salario; experiencia laboral; empleabilidad; análisis de varianza; regresión lineal.

JEL: J01, E24, C2

\section{ABSTRACT}

The study focused on the results obtained through the Labor Skills Certification service provided by the Impulsa Peru Program of the Ministry of Labor and Employment Promotion (MTPE) during the years 2011 - 2016, according to the regulations established by the General Directorate of Vocational Training and Labor Training (DGFPCL), as well as the guidelines of Budget Program 0116 "Improvement of Employability and Labor Insertion - PROEMPLEO". These results were measured based on two key indicators such as the level of work experience and the level of salary, which are linked to the employability of a person. For this, four samples were taken whose size per sample was 1,804 beneficiaries with certified labor competencies and compared with 1,804 non-competent beneficiaries. It could be concluded that the permanence in the labor market had significance in the analysis of variance and in two of the four samples, showing differences of 1 to 3 months. On the other hand, the salary showed significance both in the analysis of variance and for the four samples compared, with differences between S / 30 to S / 70 soles.

Keywords: Certification of labor competencies; wages; work experience; employability; variance analysis; linear regression.

JEL: J01, E24, C2 


\section{Introducción}

La política o sistema de competencias, según Tamayo (2012) data de los años ochenta, precisamente en Reino Unido es donde se inicia para luego expandirse a diversos países en el mundo, principalmente Europa y Norteamérica. Posteriormente, en América Latina, la expansión fue impulsa por Cinterfor de la Organización Internacional del Trabajo (OIT) en el año 1996 en una conferencia internacional dada en México. El propósito de esta política fue el de mejorar los sistemas de información de la oferta educativa e incrementar los niveles de competitividad y productividad. A raíz de la expansión de los sistemas de competencias, países como Colombia y Chile han logrado crear sistemas que actualmente están ampliamente consolidados. El caso de Chile, desde el año 2008, en el marco de la Ley 20.267 es que se crea el Sistema Nacional de Certificación de Competencias Laborales bajo la administración de la Comisión de dicho Sistema presidida por su Ministerio de Trabajo, Educación y Economía(ChileValora, 2018). Ello hace del sistema chileno un ejemplo a nivel de Sudamérica al haber diseñado un sistema que reúne los esfuerzos de tres sectores clave para la economía y el empleo.

En el caso del Perú, el Ministerio de Trabajo y Promoción del Empleo ha impulsado el desarrollo de la normalización y certificación de competencias laborales desde la Dirección de Formación Profesional y Capacitación Laboral (en el plano normativo) y el Programa Nacional para la Promoción de Oportunidades Laborales "Impulsa Perú" (como ejecutor). En específico, el Programa Impulsa Perú tiene como fin promover el empleo, la empleabilidad y el emprendimiento en el marco de lo establecido en el Programa Presupuestal 0116: "Mejoramiento de la Empleabilidad e Inserción Laboral - PROEMPLEO”.

Los antecedentes del Programa surgen en el año 2009, cuando se crea el Programa Especial de Reconversión Laboral (PERLAB), conocido también como Programa REVALORA PERÚ (Decreto de Urgencia Nº 021-2009 modificado por Ley $N^{\circ}$ 29516). En el año 2011, el Ministerio de Trabajo y Promoción del Empleo (MTPE), como parte del Viceministerio de Promoción del Empleo y Capacitación Laboral crea el Programa Nacional para la Promoción de Oportunidades Laborales "Vamos Perú", mediante Decreto Supremo N 016-2011-TR, el 20 de setiembre del 2011. En mayo del 2015, el Programa cambio al nombre actual, de "Impulsa Perú". 
Asimismo, el Programa Impulsa Perú cuenta con tres servicios: i) Capacitación para la Inserción Laboral, ii) Certificación de Competencias Laborales y la iii) Capacitación para el Autoempleo. Estos servicios están definidos en los modelos operacionales de PROEMPLEO, orientándose desde una perspectiva de presupuesto por resultados y siguiendo una cadena de valor público cuyo fin es la inserción laboral al mercado formal.

El servicio de Certificación de Competencias Laborales está orientado al reconocimiento formal y documentado de las competencias laborales que posee una persona, ya sea que éstas se hayan adquirido de manera informal o formal. Es decir, este servicio implica asegurar y afirmar de manera documentada las habilidades, conocimientos y destrezas aprendidas permitiendo a las personas desarrollar una tarea, en este caso un trabajo en un perfil laboral específico.

No obstante, dada la aplicabilidad práctica del servicio de Certificación de Competencias Laborales, se desarrolla un marco conceptual y teórico a fin de fundamentar académicamente su pertinencia como política activa de empleo.

Asimismo, el presente trabajo desarrolla un conjunto de hipótesis en el marco de la naturaleza del servicio de Certificación de Competencias Laborales a fin de poder contrastarlas con la información disponible con la que cuenta el Programa Impulsa Perú. Estos resultados ayudarán a visualizar de manera aproximada si el servicio está obteniendo resultados o requiere de ciertos ajustes en su diseño.

Finalmente, se propone determinar qué factores están relacionados con las principales variables ligadas al servicio de Certificación de Competencias Laborales, como son el nivel de salario y la permanencia en el mercado laboral de los beneficiarios.

\section{Perspectivas de la certificación de competencias laborales}

La certificación de competencias laborales ha resultado una política ampliamente difundida por la Organización Internacional del Trabajo (Vargas, 2012), así como por la Organización para la Cooperación y el Desarrollo Económico (OCDE, 2008). Por ello, países como México, Colombia y Chile han optado por implementar sistemas de certificación 
de competencias laborales a través del establecimiento de normas de competencias, así como de estándares en perfiles laborales ${ }^{3}$ y centros certificadores(Mertens, 1996). En el Perú, se viene aplicando esta política a través del Ministerio de Trabajo y Promoción del Empleo (MTPE).

¿Pero qué se entiende por certificar las competencias laborales? En primer lugar, para poder responder a esta pregunta habría que ir por partes y definir uno de sus componentes principales: las competencias laborales. Para la OCDE(Schleider \& Caddy, 2016, pág. 4), estas se definen como el "conjunto de conocimientos, habilidades y destrezas que pueden aprenderse y que permiten a los individuos desarrollar una actividad o tarea de manera adecuada y sistemática, y que pueden adquirirse y ampliarse a través del aprendizaje". Entonces, se puede decir que el grupo de competencias disponibles y distribuidas en la PEA, conforma el capital humano del país.

Por otro lado, la Real Academia Española, define a la certificación como la acción y efecto de certificar, pero también como un documento en que se asegura la verdad de un hecho. Esto va en consonancia con el término certificar, que significa asegurar, afirmar o dar por cierto algo.

De ambas definiciones, se puede decir que la certificación de competencias laborales implica asegurar y afirmar de manera documentada las habilidades, conocimientos y destrezas aprendidas permitiendo a las personas desarrollar una tarea, en este caso un trabajo en un perfil laboral específico.

Comprendiendo la definición respecto al servicio bajo análisis, cabe hacer la pregunta sobre ¿para qué le es útil la certificación de competencias laborales al mercado laboral (ofertantes y demandantes)? La respuesta a esta pregunta implica dos elementos que de alguna forma están relacionados y que implican la visibilidad de las competencias laborales y la valorización de dichas competencias laborales para ser adquiridas.

En primer lugar, la certificación en sí, de acuerdo a cómo se la ha definido, no está ligada a mejorar la productividad de los trabajadores, sino que su primer efecto es hacer visible las competencias laborales con las que ya cuenta el trabajador. Es decir, el trabajador contaba con ciertas habilidades y competencias, pero no podía demostrarlas o ponerlas de ma- 
nera explícita para un posible empleador, o para que sean valoradas en el mercado laboral. Desde esta aproximación, la certificación de competencias laborales representa un proceso orientado a reducir la asimetría de información.

En segundo lugar, cuando las competencias laborales tienen un reconocimiento, éstas alcanzan un valor para el mercado laboral, por lo que los incentivos para adquirir dichas competencias aumentan. En definitiva, la literatura indica que tener la certificación mejora la proyección laboral de una persona y orienta a otras, que aún no tienen dicha competencia, a capacitarse en ella. De ambas premisas resulta que la certificación motiva la capacitación laboral. Por ello, la confluencia de ambas estrategias tiene por efecto mejorar los niveles de productividad laboral, y por ende de la productividad de la economía.

Entonces, se puede concluir que, por sí misma, la certificación no implica necesariamente el aumento de la productividad de los trabajadores, a menos que se encuentre ligada con los procesos de capacitación tanto en el marco de los servicios públicos como los incentivos privados (Olivera Vidal, Corvalán, Ortega, Loyola, \& Illanca, 2017).

Una aproximación más amplia para responder a la pregunta planteada implica poner sobre la mesa el hecho de que la certificación de competencias laborales ayuda a visibilizar la productividad (no necesariamente aumentarla) por lo que, su efecto inmediato es la reducción en los tiempos de búsqueda de empleo. Este caso es aplicado para los trabajadores desempleados y para aquellos independientes. Sin embargo, en el caso de los trabajadores que ya cuentan con un empleo, la certificación resulta una herramienta importante para lograr su movilidad dentro de la empresa o hacia otra empresa, así como, posibles mejoras salariales. Resulta importante considerar que, la certificación expresa un reconocimiento social y, por lo tanto, puede generar un impacto importante a nivel subjetivo para el trabajador: la sociedad valora su competencia y su dignidad laboral. Otro efecto importante, cuando se da la simbiosis certificación-capacitación, es el desarrollo de itinerarios formativos para proyectar el futuro laboral de una manera más certera(Álvarez Medina \& De la O Pérez, 2005).

Desde el lado de la demanda laboral, las empresas se ven beneficiadas por la certificación dado que reduce los costos de transacción, los de 
búsqueda y contratación de trabajadores calificados. Además, la certificación permite tener un conjunto de competencias en la empresa que cumplan estándares de desempeño exigidos en el mercado. La certificación permite a la empresa desarrollar sus planes de desarrollo de su personal. Con ello reconocer a los trabajadores con mayor productividad y competitividad (Bertrand, 1997).

Sin embargo, se ha hablado ampliamente de la certificación como tal, pero no de las competencias laborales. De acuerdo a Escobar (2005, pág. 36), las competencias laborales implican el "conjunto de comportamientos socio-afectivos y habilidades cognoscitivas, psicológicas, sensoriales y motoras que permiten llevar a cabo de manera adecuada un papel, una función, una actividad o tarea".

En la actual configuración social y de economías globalizadas, el enfoque de trabajo está ceñido hacia las competencias laborales. Las personas deben demostrar y visualizar sus competencias laborales para sus organizaciones a fin de acceder a otros trabajos o permanecer en el mercado laboral, lo cual decanta en una proyección profesional. Esto quiere decir que estar en armonía con las crecientes demandas laborales amplía la probabilidad de ingresar, permanecer y desarrollarse en el campo laboral (Gómez Vélez, 2012). Este concepto está muy ligado a la empleabilidad de una persona.

\section{El concepto de empleabilidad}

Un concepto importante para la presente investigación está muy ligado al tema de la certificación de competencias laborales. Este concepto se refiere a la empleabilidad. La empleabilidad tiene diferentes definiciones desde la lupa con la que sea mirada.

En primer lugar, podemos decir que la empleabilidad según la recopilación de Ronald (2004, pág. 2) ${ }^{4}$ es la posesión de un individuo de las cualidades y competencias requeridas para calzar con las necesidades cambiantes de los empleadores y los clientes y a la vez, realizar sus propias aspiraciones y potencialidades en el trabajo.

Asimismo, según la ya citada recopilación de Ronald ${ }^{5}$, la empleabilidad también es la capacidad relativa de un individuo de alcanzar empleo 
significativo dada la interacción entre las circunstancias personales y el mercado de trabajo.

Asimismo, la empleabilidad es el desarrollo del potencial, el crecimiento intelectual, la mejora de las habilidades, el entendimiento y atributos, que empoderan a las personas para realizar impactos sobre la sociedad en beneficio recíproco, la comunidad y la economía (O’Brien \& Silva, 2012).

La Organización Internacional del Trabajo (OIT) definió la empleabilidad como: "...uno de los resultados fundamentales de una educación y formación de alta calidad y de la ejecución de varias otras políticas. Abarca las calificaciones, conocimientos y las competencias que aumentan la capacidad de los trabajadores para conseguir y conservar un empleo, mejorar su trabajo y adaptarse al cambio, elegir otro empleo cuando lo deseen o pierdan el que tenían e integrarse más fácilmente en el mercado de trabajo en diferentes períodos de su vida" (Rodríguez Cuba, 2009, pág. 11). Es por ello que, para la OIT, hay dos dimensiones claras en este concepto, la capacidad personal y el resultante social. Estas dos dimensiones van a permitir que las personas puedan conseguir, conservar, cambiar, mejorar su trabajo, adaptarse al cambio social, insertarse en el mercado laboral e integrarse a la sociedad.

Estas definiciones, como se puede apreciar, involucran varios elementos que son constituyentes del concepto de empleabilidad. Se puede describir cada elemento en función a lo siguiente (Forrier \& Sels, 2003):

i. La empleabilidad implica características personales. Estas pueden ser las habilidades de una persona para conseguir o mantenerse en un trabajo.

ii. El contexto es importante dado que representa la demanda de trabajo del empleador. Se define la demanda como elemento para la movilidad o posibilidades para el empleado de ascender y crecer laboralmente. Este aspecto está ligado a la línea de carrera.

iii. Efecto de la empleabilidad es importante. En este caso, la calidad del trabajo obtenido o mantenido por la persona es un efecto de la capacidad de empleabilidad de la misma. 
iv. Las actividades desarrolladas como la capacitación, mejora de las habilidades laborales, etc. da un enfoque de orientación a la empleabilidad. Este aspecto se refiere a la actitud y la conducta del empleado hacia mejorar su empleabilidad.

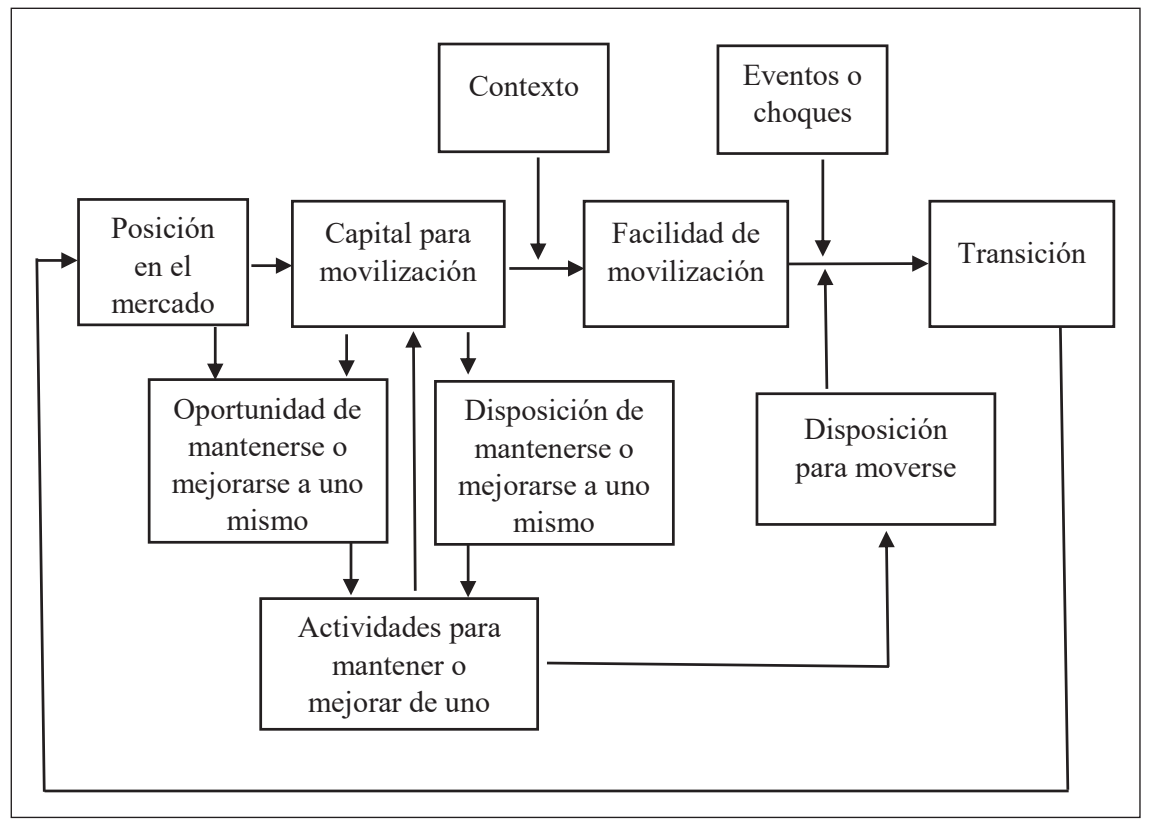

Figura 1. Modelo del proceso de empleabilidad

Fuente: Forrier y Sels (2003). The concept of employability: a complex mosaic.

Higher Institute of Labour Studies. Bélgica, pág. 108.

La figura anterior nos provee un mapa muy interesante donde se puede apreciar inputs y outputs, así como factores internos y externos que se interrelacionan mutuamente. Desde la posición en el mercado laboral hasta la transición se aprecian dos características fundamentales, como son las oportunidades y la disposición a mantenerse o mejorar respecto de la posición (en el empleo) que se tiene.

Por otro lado, algunos estudios han elaborado un marco conceptual orientado a un análisis empírico respecto a la empleabilidad. Pitan y Atiku (2017) plantearon un modelo en donde consideran a la empleabili- 
dad como su variable dependiente y al factor de toma de decisiones (planificación personal), la oportunidad de autoconciencia (compromiso con la realidad, aprendizaje basado en el trabajo) y el aprendizaje de transición (aprendizaje de desarrollo profesional) como sus variables independientes. Concluyen que las actividades de orientación profesional tienen influencia positiva en la empleabilidad de los estudiantes.

De igual manera, Herman (2015) realizó una investigación que analizó tres factores de género identificados como responsables del resultado sobre género, normalidad de roles, localidad y movilidad, y barreras estructurales e institucionales. Los factores incluidos en su modelo conceptual respecto a la empleabilidad incluyen elementos individuales, personales y externos. Entre los individuales incluidos son los atributos esenciales, competencias personales, habilidades transferibles, cualificaciones, experiencia laboral, características demográficas (edad, género, etnicidad, etc.), salud, discapacidad, habilidades para entrevistas laborales, $\mathrm{CV}$, conciencia del mercado de trabajo, adaptabilidad y movilidad (geográfica y ocupacional). Entre los factores personales incluidos son las circunstancias del hogar, cultura de trabajo familiar, acceso a capital financiero y capital social. Finalmente, entre los factores externos se incluyeron los factores del mercado laboral, factores macroeconómicos, prácticas de reclutamiento, características de las vacantes y factores de política de empleo.

\section{Modelo de la certificación de competencias laborales}

Si bien se ha referido toda la connotación social que implica la certificación de competencias laborales, para poder plantear un estudio sobre los resultados es necesario observar qué modelos han sido planteados para entender, de manera teórica, los efectos de la certificación y sus ventajas.

Como ya se ha indicado, la certificación representa el reconocimiento de la competencia laboral que posee el trabajador. En ese sentido, expresa una señal oficial, formal y documentada que puede ser valorada en el mercado laboral por el empleador. Esta aproximación de la certificación de competencias laborales está ligada al "Modelo de Señalización en el Mercado de Trabajo", desarrollado por Michael Spence ${ }^{6}$. 
En su artículo, Spence(1973), busca conceptualizar un enfoque en donde el poder de señalización de la educación, la experiencia laboral, cuestiones como la raza, el sexo u otras características personales observables pueden ser determinantes en el mercado laboral. Como se aprecia, él ya considera en su modelo la experiencia laboral.

El modelo parte por la siguiente premisa: el proceso de contratación de un trabajador es una decisión de inversión para una empresa. Pero también, el hecho de promover a un trabajador ya contratado. El hecho de que el empleador no conozca las habilidades del trabajador hace que dicha decisión este bajo un marco de incertidumbre. Otra premisa que se usa en el modelo se refiere a que el término señal es usado solamente para aquellas características que pueden ser cambiadas por el trabajador, como la educación o la experiencia laboral.

Spence (1973) establece un bucle informacional en el mercado de trabajo. Este bucle, tiene como primer paso las expectativas probabilísticas condicionadas del empleador en función a las señales e índices ${ }^{7}$. Estas expectativas le ayudan a definir un nivel de salario a ofrecer. Las personas que aplican al trabajo asumen los costos de señalización tratando de maximizar el retorno de estos. El paso siguiente implica la contratación y la consecuente observación de la productividad marginal del empleado y sus señales informadas. La brecha que pueda surgir entre las expectativas del empleador y la observación de las habilidades del empleado modifican las expectativas probabilísticas condicionadas y se origina un proceso de aprendizaje(Cahuc \& Zylberberg , 2001).

En el proceso de contratación y la promoción de un trabajador, el empleador se enfrentará al problema de la selección adversa. Partiendo por el hecho de que en el mercado laboral hay dos tipos de trabajadores: una proporción de ellos con productividad alta y otra con productividad baja, es de esperar que los empleadores no puedan identificarlos. El salario pagado será aquel igual a la productividad laboral esperada para cada trabajador. Pero este equilibrio contractual es ineficiente dado que los trabajadores con alta productividad están subsidiando a los de productividad baja. Entonces, los trabajadores que inviertan más en la señalización de sus habilidades podrán diferenciarse y hacer que los empleadores reconozcan y acepten esta señal para ofrecer mayor nivel de salario, o que 
sean promovidos manteniéndose en otro puesto de trabajo(Acemoglu \& Autor, 2015).

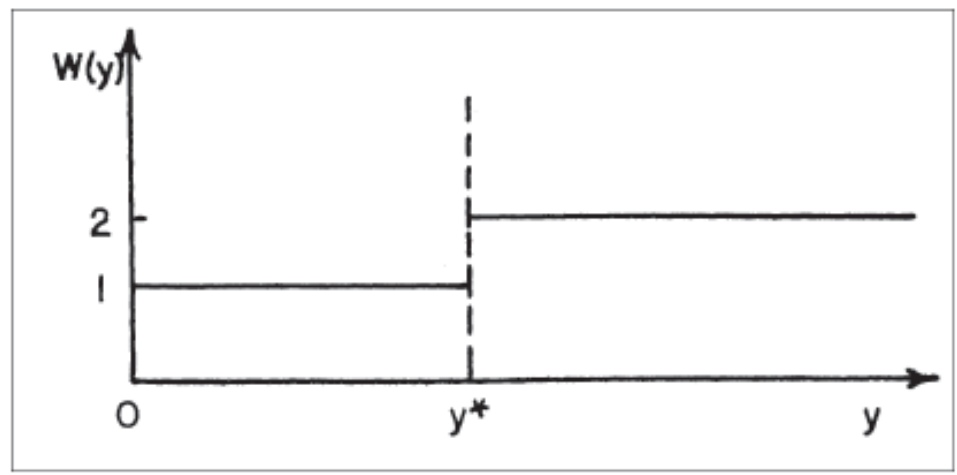

Figura 2. Salarios ofrecidos como función del nivel de educación Fuente: Spence, M. (1973). Job Market Signaling. The Quaterly Journal of Economics, 335-374.

Resulta posible aproximar el efecto de la certificación de competencias laborales a partir de la Figura 2 propuesto por Spence donde se visualiza el eje $W(y)$, que represente el salario ofrecido, y el eje $y$ que es el índice que mide el nivel de educación. El salto del salario 1 a 2 implica el reconocimiento de un mayor nivel educativo en el trabajador. Si cambiamos la variable $y$, como la certificación de competencias laborales, podemos ver que aquellas personas que logran aprobar la evaluación tienen un mecanismo para que el empleador reconozca su productividad. Este reconocimiento cambia el estatus del trabajador hacia aquellos con productividad 2 , generando que reciban una oferta de salario de valor 2. Asimismo, la certificación de competencias laborales puede ayudar a que un trabajador sea promovido, lo que implica que se le reconozca un salario de nivel 2, cuando antes se le pagaba el de nivel 1.

\section{Análisis de datos y resultados}

Para el presente estudio la fuente de datos fue brindada por el Programa Impulsa Perú de acuerdo al registro del mismo (SISREG), la cual corresponde a información pública durante el periodo 2011-2016 a nivel nacional, anonimizada de 18,744 beneficiarios correspondientes a la población 
objetivo del servicio, es decir personas con vulnerabilidad sociolaboral cuyas características son similares en cuanto a su inclusión bajo los criterios de focalización específicos descritos en el Programa Presupuestal 0116 PROEMPLEO.

Asimismo, se tomó cuatro muestras aleatorias del total de grupo de 18,744 a fin de comparar al grupo de personas que lograron salir competentes laboralmente con el grupo que resultó no competente en la evaluación para la certificación de competencias laborales. Estas muestras se identificaron mediante el muestro aleatorio simple.

\subsection{Resultado de la certificación de competencias laborales sobre el nivel de experiencia laboral}

Un primer planteamiento que se dedujo de las perspectivas teóricas de la certificación de competencias laborales, implica que éste, puede repercutir sobre la permanencia en el mercado laboral. Es decir, si una persona logra demostrar sus habilidades y competencias laborales de manera certificada, entonces posee una herramienta que puede hacer notar alguno de los componentes de su empleabilidad.

El análisis de datos de los beneficiarios ha mostrado una tendencia interesante que puede ayudar a comprender el resultado de la certificación de competencias laborales sobre el nivel de experiencia laboral. La Figura 3 muestra la densidad de Kernel (estimada) del tiempo laboral registrado en la planilla electrónica por parte de los beneficiarios que se certificaron antes de haber ingresado a este registro. Es decir que la gráfica muestra a aquellos que recibieron el tratamiento (el servicio de certificación) y que luego han tenido registros de contratos formales en la planilla electrónica. Esta densidad ha sido diferenciada según el resultado de la evaluaciónpor muestra analizada. Se han tomado cuatro muestras de igual tamaño. La razón de ello es que las personas que resultaron no competencias laboralmente (en el perfil evaluado) equivalen a 1804 personas, mientras que las personas que sí resultaron competentes laboralmente equivale a más de 16,000 personas. La comparación directa puede hacer que tengamos sesgos e inexactitudes. Es por ello que se consideró tomar cuatro muestras aleatorias de igual tamaño para compararlas una por una. 
Como bien se ha dicho, hay dos resultados posibles: i) competente laboralmante y ii) no competente laboralmente. La densidad de Kernel de las personas que resultaron no competentes tiende a ubicarse hacia la izquierda (línea sólida azul), mientras que la densidad de Kernel de las personas que resultaron competentes laboralmente tiende a ubicarse hacia la derecha (línea punteada roja). El mismo patrón se observa para las cuatro muestras escogidas.

Este resultado es revelador, dado que representa un indicio de que las personas que lograron culminar con éxito el proceso de certificación de competencias laborales han incremantado su permanencia en el mercado laboral. El eje X de la gráfica mide el tiempo laboral (plazos de contrato en la planilla electrónica) en años, el movimiento a la derecha quiere decir que el promedio de años trabajando se ha incrementado. El mismo resultado mostrado para las cuatro muestras aleatorios nos sugiere que esta diferencia es significativa y hay que considerarla pertiente.

Sin embargo, si bien usando el criterio gráfico se puede brindar una primera conclusión es importante considerar un test estadístico que nos permita asegurar (con un margen estadístico de error) la hipótesis planteada. Para ello, la Tabla 1 muestra la estadísticas descriptivas de la variable "experiencia laboral (plazo del contratos en la planilla electrónica)" diferenciadas según el resultado de evaluaciónpor muestra aleatoria tomada.

Lo que se puede apreciar es que, en promedio, las personas que resultaron compententes laboralmente en el perfil evaluado han permanecido en los registros de la planilla electrónica entre 3.04y 3.17años, mientras que las personas que no resultaron competentes laboralmente en el perfil evaluado alcanzaron un valor promediode 2.89 años. La máxima diferencia equivale a alrededor de 3 meses y 10 días, mientras que la mínima diferencia equivale a 1 mes y 24 días.

Asimismo, vemos que la variabilidad de los grupos es muy similar a pesar de la diferencia en el número de personas en cada condición, salvo para el caso de la mínima y máxima diferencia. El total de las personas, se muestra que han permanecido en promedio un máximo de 3.02 años y un mímo de 2.96 años. 


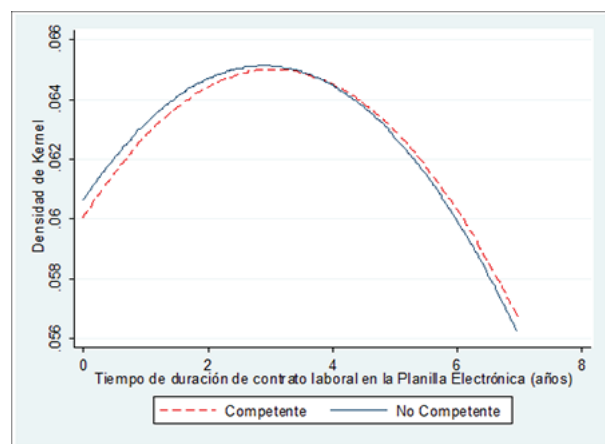

(a) Muestra 1

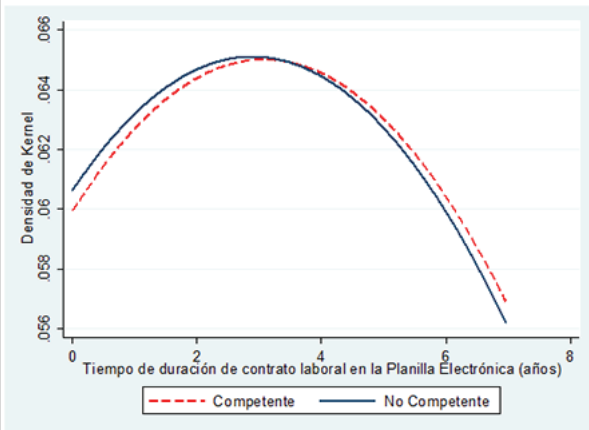

(c) Muestra 3

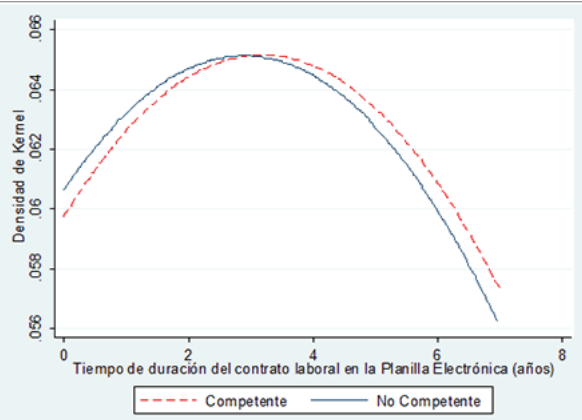

(b) Muestra 2

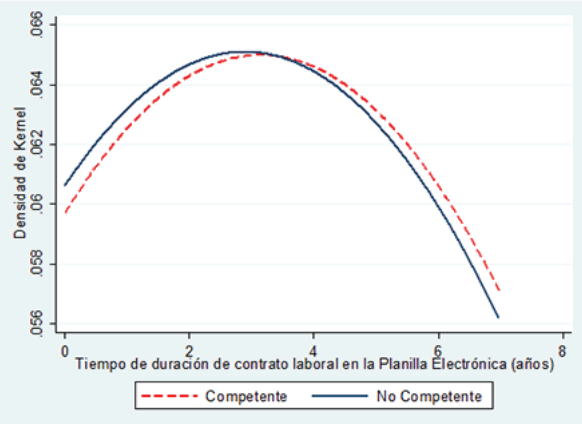

(d) Muestra 4

Figura 3. Densidad de Kernel de la experiencia laboral del beneficiario según plazo de contrato (años), diferenciado por el resultado de evaluación y por la muestra tomada.

Fuente: Elaboración propia en base a la información pública del Programa Impulsa Perú (MTPE) y la Planilla Electrónica

Tabla 1

Estadísticas de la experiencia laboral anual según resultado de evaluación

\begin{tabular}{|l|c|c|c|c|}
\hline Resultado final & Muestra 1 & Muestra 2 & Muestra 3 & Muestra 4 \\
\hline Competente & 3.04 & 3.17 & 3.07 & 3.14 \\
\hline No Competente & 2.89 & 2.89 & 2.89 & 2.89 \\
\hline Total & 2.96 & 3.02 & 2.98 & 3.01 \\
\hline
\end{tabular}

Fuente: Elaboración propia en base a la información pública del Programa Impulsa Perú (MTPE) y la Planilla Electrónica

Sin embargo, las diferencias calculadas solamente representan un valor aritmético, pero a nivel de la estadística se debe de probar si este valor aritmético es significativo para todas las muestras aleatorias. 
Para ello se procedió a realizar un análisis de varianza a fin de determinar si existe una diferencia estadística. La Tabla 2 muestra el test $\mathrm{F}$ de dicho análisis respectivamente. El valor del estadístico F oscila entre 15.62 como máximo y 4.13 como mínimo. Estos valores nos dan probabilidades menores a 0.05 para cada muestra aleatorio. Este resultado nos dice que efectivamente existe una diferencia estadística significativa en cuanto al nivel de experiencia laboral (plazo del contratos en la planilla electrónica) entre las personas competentes y no competentes laboralmente considerando cada muestra.

Tabla 2

Análisis de varianza de la experiencia laboral

\begin{tabular}{|l|c|c|c|c|}
\hline \multirow{2}{*}{ Estadístico } & \multicolumn{2}{|c|}{ Test F } & \multicolumn{2}{c|}{ Test de Bartlett } \\
\cline { 2 - 5 } & F & Prob. & Chi2 & 0.248 \\
\hline Muestra 1 & 4.13 & 0.0421 & 1.3360 & 0.788 \\
\hline Muestra 2 & 15.62 & 0.0001 & 0.0721 & 0.366 \\
\hline Muestra 3 & 6.68 & 0.0098 & 0.8181 & 0.307 \\
\hline Muestra 4 & 11.86 & 0.0006 & 1.0434 & \\
\hline
\end{tabular}

Fuente: Elaboración propia en base a la información pública del Programa Impulsa Perú (MTPE) y la Planilla Electrónica

Este primer resultado nos arroja que lo obtenido en la evaluación para la Certificación de Competencias Laborales marca una diferencia significativa en cuanto a lograr que las personas que competentes laboralmente en el perfil evaluado puedan permanecer en el mercado laboral formal (registrados en planilla electrónica) en promedio entre 1.5 y 3.2 meses más que aquellas que no resultaron competentes laboralmente en el perfil evaluado. En el entorno de los grupos poblacionales que atiende el Programa, para una persona en condición de desempleo o subempleo la posibilidad de trabajar este tiempo adicional es muy valorado.

\subsection{Resultado de la certificación de competencias laborales sobre el nivel de salario}

Un segundo planteamiento, mostrado en el modelo de Spencer, implica que la certificación de competencias laborales representa un mecanismo de señal para el mercado laboral. Es decir, si una persona logra demostrar sus habilidades y competencias laborales de manera certificada y docu- 
mentada, entonces posee una herramienta que puede hacer notar alguno de los componentes de su empleabilidad a fin de mejorar o tener incidencia sobre nivel de su salario.

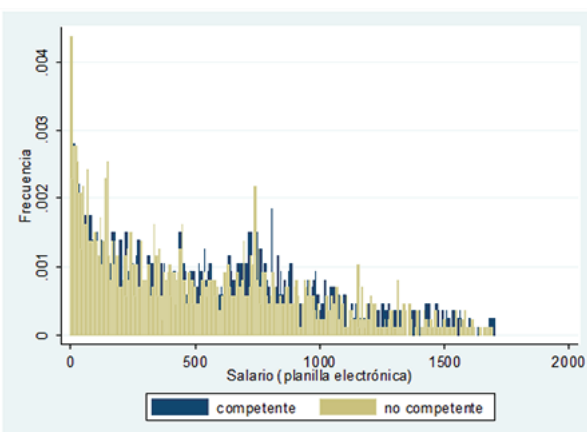

(a) Muestra 1

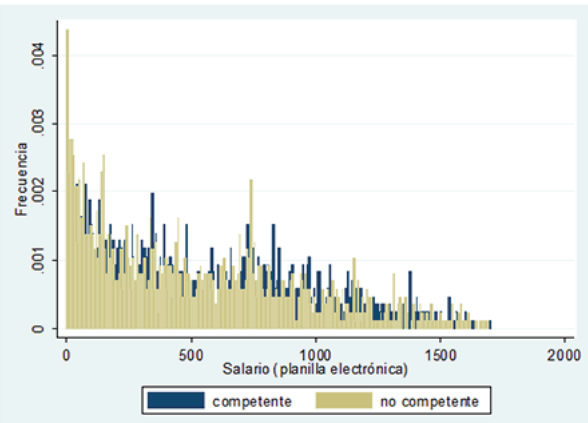

(c) Muestra 3

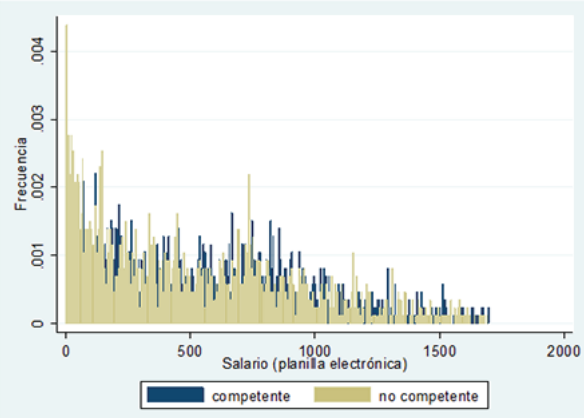

(b) Muestra 2

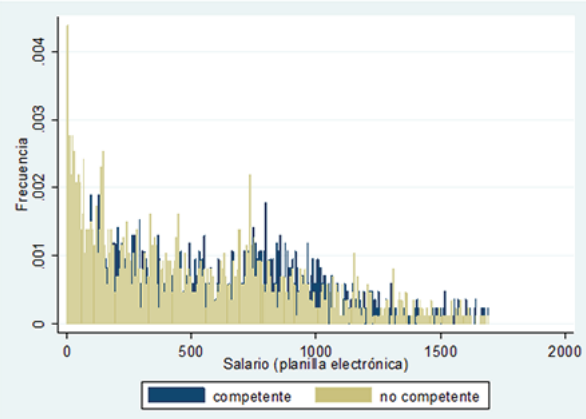

(d) Muestra 4

Figura 4. Histograma del Salario Promedio (2011-2016)

Fuente: Elaboración propia en base a la información pública del Programa Impulsa Perú (MTPE) y la Planilla Electrónica

Una primera aproximación visual mostrada en la Figura 4 nos indica que el histograma de las personas competentes laboralmente en el perfil evaluado, tiene mayores frecuencias en salarios más altos, mientras que el histograma de las personas que resultaron no competentes laboralmente en el perfil evaluado tiene mayores frecuencias en salarios menores. Este mismo patrón se repite para cada una de las muestras aleatorias tomadas. Esta diferencia en los histogramas de cada grupo da un primer indicio de que el resultado del servicio puede repercutir sobre el nivel de salario del beneficiario certificado en sus competencias laborales. 
Este indicio puede ser fácilmente corroborado cuando se analiza la evolución de los salarios de los beneficiarios del servicio registrados en la planilla electrónica. De acuerdo a la Figura 5, las líneas sólidas muestran la tendencia de los salarios de las personas que resultaron competentes laboralmente diferencias por cada muestra aleatoria. Por otro lado, la línea punteada muestra la tendencia de los salarios de las personas que resultaron no competentes laboralmente. Como se puede apreciar existe una brecha entre los niveles salariales que van ampliándose a partir del inicio del periodo de análisis (año 2011). Para el año 2015 se aprecia ligeramente que la brecha es mayor que los años anteriores. Sin embargo, hay un quiebre en el primer trimestre del año 2016. Esto se puede deber a la forma de medición de los registros de la planilla electrónica con el cruce de los beneficiarios. Para fines del año 2016 se ha tenido menores registros cruzados.

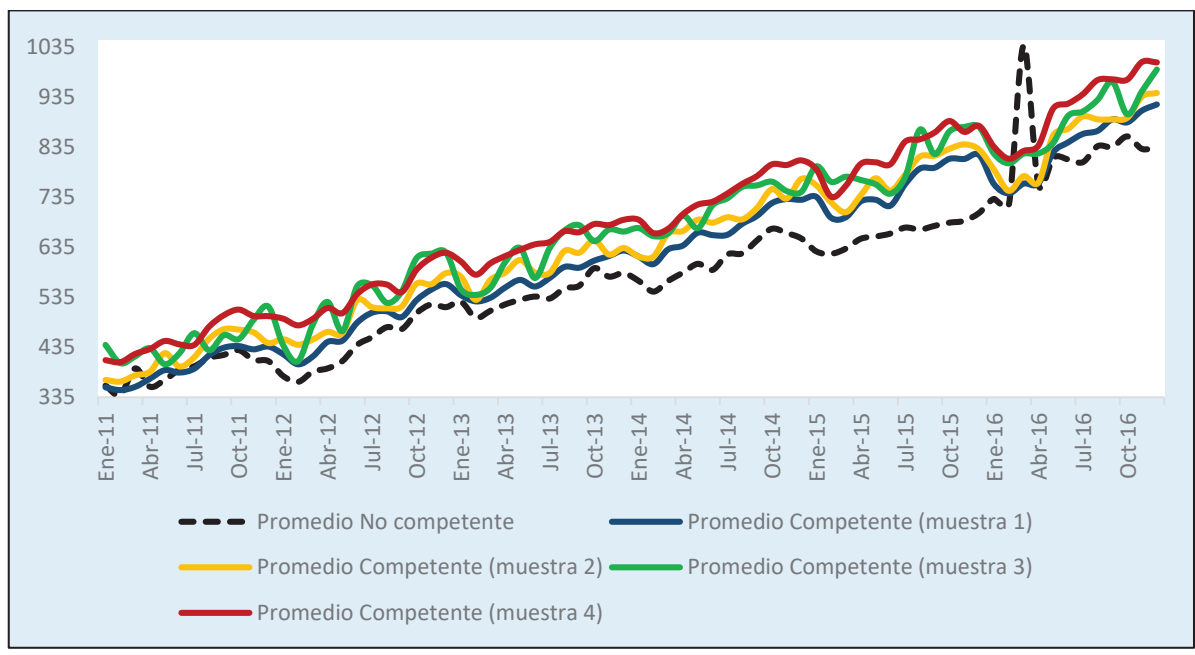

Figura 5. Evolución de los salarios promedio de los beneficiarios, registros de planilla electrónica, según resultado de la evaluación

Fuente: Elaboración propia en base a la información pública del Programa Impulsa Perú (MTPE) y la Planilla Electrónica

La visualización de la tendencia de ambos salarios durante el periodo de análisis plantea la hipótesis de que ambos grupos de personas han tenido desempeños en el nivel de salarial marcadamente diferentes. Sin 
embargo, como se ha mencionado anteriormente, considerar la diferencia aritmética no es garantía de una diferencia estadística.

Para ello se plantea el uso del análisis de varianza a fin de estimar la significancia de las diferencias en cada periodo de análisis y por cada muestra aleatoria tomada. La Figura 6 muestra la tendencia del Test F que ha sido calculado para cada periodo (línea sólida). Asimismo, se muestra la probabilidad correspondiente a cada test F. lo que se puede apreciar es que al inicio del periodo de análisis la diferencia entre los salarios por cada grupo no es significativa. Durante todo el año 2011 no se aprecia diferencia entre los niveles de salario. Sin embargo, a partir del año 2012 se puede ver que el test $\mathrm{F}$ marca valores altos con probabilidades de 0.00 , lo cual permite concluir que las diferencias son estadísticas. Por otro lado, para el año 2016, en los meses de abril y mayo se ve que hay una tendencia a que los salarios se igualen, pero está vuelve a diferenciarse a partir de junio hasta fin del año 2016.

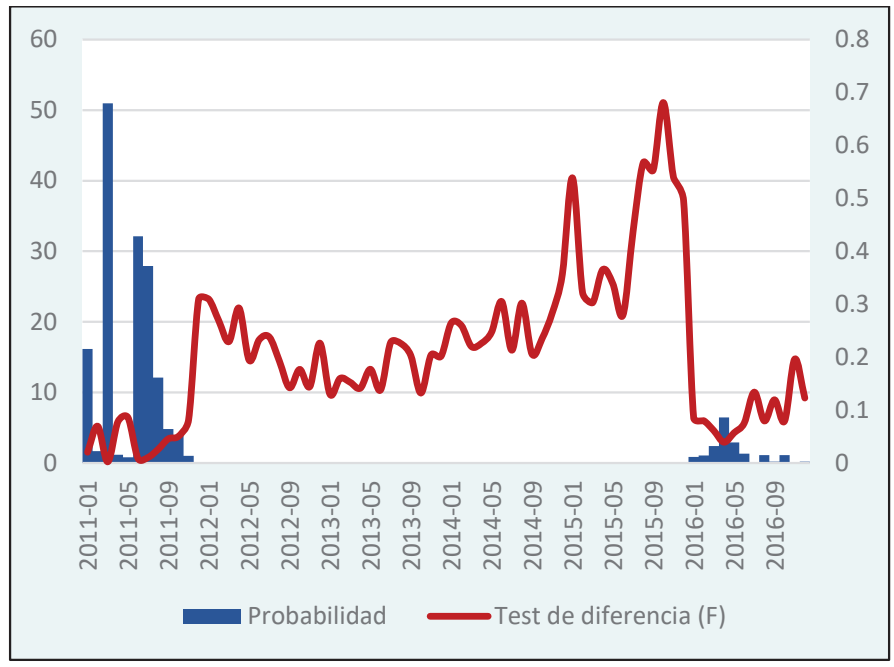

Figura 6. Evolución del Test de diferencia (análisis de varianza) para los salarios entre personas competentes y no competentes en su evaluación de competencias laborales 
La Tabla 3 muestra las estadísticas de la variable nivel de salario diferenciados según las personas competentes y no competentes durante todo el periodo de análisis (2011-2016) y separadas por cada muestra aleatoria escogida. Como se puede apreciar, para el caso de las personas que resultaron competentes laboralmente su salario promedio durante el periodo tuvo como un máximo el valor de S/ 569.32 y un mínimo de S/ 536.39, mientras que las personas que resultaron no competentes laboralmente tuvieron un nivel de salario de S/ 503.55. La máxima diferencia encontrada es de S/ 65.89, mientras que la mínima diferencia es de S/ 32.84 .

Tabla 3

Estadísticas descriptivas del nivel de salario $(\mathrm{S} / \mathrm{)}$

\begin{tabular}{|l|c|c|c|c|}
\hline Resultado final & Muestra 1 & Muestra 2 & Muestra 3 & Muestra 4 \\
\hline Competente & 541.04 & 561.52 & 536.39 & 569.32 \\
\hline No Competente & 503.55 & 503.55 & 503.55 & 503.55 \\
\hline Promedio & 522.23 & 532.43 & 519.39 & 536.03 \\
\hline Diferencia & 37.49 & 57.97 & 32.84 & 65.77 \\
\hline
\end{tabular}

Fuente: Elaboración propia en base a la información pública del Programa Impulsa Perú (MTPE) y la Planilla Electrónica

Para poder demostrar que la diferencia del nivel de salarios es significativa, se procedió a realizar un análisis de varianza tal y como se planteó anteriormente. De acuerdo a la Tabla 4 se aprecia que el estadístico F tiene un valor máximo de 21.4 y un valor mínimo de 5.55 , con probabilidades menores a 0.05 . Este resultado indica que la diferencia de salarios durante el periodo entre los dos grupos de personas (y por muestra) es significativa estadísticamente.

Por otro lado, también se ha considerado el Test de Bartlett. Este test prueba que las muestras provienen de poblaciones con la misma varianza, es decir presenta homocedasticidad. El valor de las probabilidades calculadas para este indicador por cada muestra es mayor a 0.05 , por lo cual se acepta la hipótesis nula de igualdad de varianzas entre los grupos escogidos.

Este segundo resultado nos arroja que el servicio de Certificación de Competencias Laborales ha tenido un impacto significativo en cuanto a lograr que las personas que resultaron competentes laboralmente tengan un nivel de salario mayor (registrados en planilla electrónica) 
en promedio entre S/ 68 y S/ 32 más que aquellas que no resultaron competentes laboralmente. En el entorno de los grupos poblacionales que atiende el Programa, para una persona en condición de desempleo o subempleo la posibilidad de mejorar sus ingresos en un valor promedio de S/ 50 más resulta de vital importancia y pertinencia.

Tabla 4

Análisis de Varianza del nivel de salario

\begin{tabular}{|l|c|c|c|c|}
\hline \multirow{2}{*}{ Estadístico } & \multicolumn{2}{|c|}{ Test F } & \multicolumn{2}{c|}{ Test de Bartlett } \\
\cline { 2 - 5 } & F & Prob. & Chi2 & Prob. \\
\hline Muestra 1 & 7.14 & 0.0076 & 0.0410 & 0.840 \\
\hline Muestra 2 & 17.01 & 0.0000 & 0.1068 & 0.744 \\
\hline Muestra 3 & 5.55 & 0.0185 & 0.3307 & 0.565 \\
\hline Muestra 4 & 21.40 & 0.0000 & 0.8336 & 0.361 \\
\hline
\end{tabular}

Fuente: Elaboración propia en base a la información pública del Programa Impulsa Perú (MTPE) y la Planilla Electrónica

\subsection{Factores relacionados al resultado del servicio de certifica- ción de competencias laborales}

En los numerales anteriores, se ha podido indagar que la certificación de competencias laborales ha tenido un resultado de mejor desempeño para aquellos beneficiarios que resultaron competentes laboralmente. Este grupo de beneficiarios se ha diferenciado en sus niveles de salario y de permanencia en el mercado laboral a través del registro de los plazos de sus contratos en la planilla electrónica. Se pudo probar ambas hipótesis de manera estadística.

Sin embargo, en esta sección se buscará determinar qué factores están relacionados con las dos variables más importantes, como son el nivel de salario y el tiempo de permanencia en el mercado laboral.

En primer lugar, partiendo del concepto de empleabilidad como "... uno de los resultados fundamentales de una educación y formación de alta calidad y de la ejecución de varias otras políticas. Abarca las calificaciones, conocimientos y las competencias que aumentan la capacidad de los trabajadores para conseguir y conservar un empleo, mejorar su trabajo y adaptarse al cambio, elegir otro empleo cuando lo deseen o pierdan el que tenían e integrarse más fácilmente en el mercado de trabajo en diferentes períodos de su 
vida", se puede tomar en cuenta dos elementos centrales, como son la de conseguir o mejorar, y conservar un empleo. Estos dos elementos pueden ser cuantificados por el nivel de salario y el nivel de permanencia en el mercado laboral.

Por otro lado, siguiendo a Pitan, Atiku (2017) y Herman (2015) se plantea a la empleabilidad como una variable dependiente de factores que pueden ser personales, individuales y de política. Estos factores representan las variables independientes.

De acuerdo a la disponibilidad de los datos se ha propuesta la siguiente matriz en donde se visualizan las variables a tomarse en cuenta para plantear dos modelos en estudio:

Tabla 5

Planteamiento delos Modelos de Empleabilidad

\begin{tabular}{|c|c|c|c|}
\hline \multicolumn{3}{|c|}{ Variables Independientes } & Variable Dependiente \\
\hline Factores Individuales & Factores Personales & Factores de política & Empleabilidad \\
\hline $\begin{array}{l}\text { - Sexo } \\
\text { - Edad } \\
\text { - Ámbito Geográfico }\end{array}$ & $\begin{array}{l}\text { - Educación } \\
\text { - Estado laboral } \\
\text { - Tipo de trabajador }\end{array}$ & $\begin{array}{l}\text { - } \text { Monto del convenio } \\
\text { - } \text { Fuente de financiamiento } \\
\text { - } \text { Resultado de Evaluación } \\
\text { - Nivel de Salario }\end{array}$ & $\begin{array}{l}\text { - Tiempo de perma- } \\
\text { nencia en el mercado } \\
\text { laboral, medido por el } \\
\text { registro de contratos } \\
\text { en la planilla elec- } \\
\text { trónica }\end{array}$ \\
\hline $\begin{array}{l}\text { - Sexo } \\
\text { - Edad } \\
\text { - Ámbito Geográfico }\end{array}$ & $\begin{array}{l}\text { - Educación } \\
\text { - Estado laboral } \\
\text { - Tipo de trabajador }\end{array}$ & $\begin{array}{l}\text { - } \text { Monto del convenio } \\
\text { - } \text { Fuente de financiamiento } \\
\text { - } \text { Resultado de Evaluación } \\
\text { el mercado laboral, medido } \\
\text { por el registro de contratos } \\
\text { en la planilla electrónica }\end{array}$ & - Nivel de Salario \\
\hline
\end{tabular}

Fuente: Elaboración propia

Una vez identificados los componentes del modelo de acuerdo a la disponibilidad de los datos es importante realizar un análisis por cada indicador que se ha postulado para medir la empleabilidad.

En primer lugar, se parte por el indicador de tiempo de permanencia en el mercado laboral, medido por el registro de contratos en la planilla electrónica. 
Tabla 6

Regresión lineal (MCO) de la experiencia laboral

\begin{tabular}{|lccccccccc|}
\hline \multirow{2}{*}{$\begin{array}{c}\text { Experiencia } \\
\text { (plazos contratos) }\end{array}$} & \multicolumn{2}{c}{ Muestra 1 } & \multicolumn{2}{c}{ Muestra 2 } & \multicolumn{2}{c|}{ Muestra 3 } & \multicolumn{2}{c|}{ Muestra 4 } \\
\cline { 2 - 9 } & Coef. & P>t & Coef. & P>t & Coef. & P>t & Coef. & P>t \\
\hline Salario & 0.0024 & 0.0000 & 0.0024 & 0.0000 & 0.0024 & 0.0000 & 0.0025 & 0.0000 \\
Edad & 0.0141 & 0.0000 & 0.0134 & 0.0000 & 0.0134 & 0.0000 & 0.0133 & 0.0000 \\
Educación & -0.0878 & 0.0850 & -0.1353 & 0.0060 & -0.1417 & 0.0060 & -0.0950 & 0.0640 \\
Resultado de Evaluación & 0.0871 & 0.1720 & 0.1842 & 0.0040 & 0.1271 & 0.0490 & 0.1110 & 0.0830 \\
Estado Laboral & 0.1377 & 0.0010 & 0.1136 & 0.0060 & 0.1003 & 0.0160 & 0.1214 & 0.0040 \\
Constante & 1.1030 & 0.0000 & 1.3501 & 0.0000 & 1.3938 & 0.0000 & 1.1920 & 0.0000 \\
\hline
\end{tabular}

Fuente: Elaboración propia en base a la información pública del Programa Impulsa Perú (MTPE) y la Planilla Electrónica

De acuerdo al modelo presentado en la Tabla 6 se ha considerado solamente las variables que resultaron significativas: Salario, Educación, Estado Laboral y Resultado de la Evaluación de certificación de competencias laborales. Los modelos estimados tienen significancia en su conjunto dado que los estadísticos F fueron de 150.5, 146, 141.64 y 152.89para cada muestra con una probabilidad de valor cero ( $p$ value igual a cero) respectivamente. Asimismo, se aprecia que los parámetros estimados son significativos con probabilidades p values iguales a cero; a excepción de dos casos como son el nivel educativo y el resultado de evaluación los cuales no fueron significativos para la muestra 1 y la muestra 4 .

La interpretación del modelo según los parámetros estimados y sus respectivas variables se detalla a continuación:

- Hay una correlación positiva entre el plazo del registro de contratos en la planilla electrónica y el nivel de salario, la edad de la persona, el resultado de evaluación y el estado laboral. Esto quiere decir que cuando una persona tiene mayores salarios, posiblemente esté dispuesta a permanecer más tiempo en el trabajo. Asimismo, cuando la persona tiene más edad acumula mayor experiencia laboral. Además, cuando la persona resulta competente laboralmente tiene un número mayor del plazo de contratos registrado en la planilla electrónica. También, cuando la persona está pasa de ser desempleado a subempleado, entonces ha tenido mayores registros de contratos en la planilla electrónica. Finalmente, si la 
persona resulta competente laboralmente, entonces tiende a tener más registros de contratos en la planilla electrónica. Sin embargo, esta última variable no resulta significativa en dos de las muestras tomadas.

- Por otro lado, hay una correlación negativa entre el nivel educativo y el registro de contratos en la planilla electrónica. Sin embargo, este variable resultó no significativa para dos muestras.

Siguiendo la misma lógica, se procedió a estimar el modelo de empleabilidad, pero usando la variable del nivel de salario. De acuerdo a la Tabla 7, el modelo tiene significancia global con un estadístico F de 93.17, $87.25,83.64$ y 105.68 respectivamentey una probabilidad de 0.000 . Asimismo, se obtuvieron las variables significativas Experiencia Laboral, Sexo, Edad, Educación, Resultado de Evaluación, Estado Laboral, Tipo de Trabajador, Inversión del Programa y Ámbito Geográfico.

Tabla 7

Regresión lineal (MCO) del nivel de salario

\begin{tabular}{|l|c|c|c|c|c|c|c|c|}
\hline \multirow{2}{*}{ Salario } & \multicolumn{2}{|c}{ Muestra 1 } & \multicolumn{2}{c}{ Muestra 2 } & \multicolumn{2}{c|}{ Muestra 3 } & \multicolumn{2}{c|}{ Muestra 4 } \\
\cline { 2 - 8 } & Coef. & P>t & Coef. & P>t & Coef. & P>t & Coef. & P>t \\
\hline $\begin{array}{l}\text { Experiencia (plazos } \\
\text { contratos) }\end{array}$ & 81.0391 & 0.0000 & 83.3831 & 0.0000 & 78.2058 & 0.0000 & 84.9430 & 0.0000 \\
Sexo & -113.1991 & 0.0000 & -108.1303 & 0.0000 & -92.6299 & 0.0000 & -128.0603 & 0.0000 \\
Edad & 7.0205 & 0.0000 & 6.6917 & 0.0000 & 6.7639 & 0.0000 & 7.1074 & 0.0000 \\
Educación & 37.2811 & 0.0000 & 24.7334 & 0.0170 & 27.2689 & 0.0110 & 21.3387 & 0.0450 \\
Resultado de Evaluación & 40.1711 & 0.0030 & 30.8549 & 0.0250 & 38.9687 & 0.0050 & 57.9835 & 0.0000 \\
Estado Laboral & -69.2065 & 0.0000 & -63.1914 & 0.0000 & -96.8568 & 0.0000 & -84.1654 & 0.0000 \\
Tipo de Trabajador & 251.7104 & 0.0000 & 225.6164 & 0.0000 & 304.2279 & 0.0000 & 277.6239 & 0.0000 \\
Inversión del Programa & -4.1100 & 0.0000 & -3.8900 & 0.0000 & -3.1600 & 0.0050 & -4.6200 & 0.0000 \\
Ámbito Geográfico & -139.8327 & 0.0030 & -102.0448 & 0.0420 & -128.6221 & 0.0270 & -163.1097 & 0.0000 \\
Constante & -58.4817 & 0.4240 & -43.8295 & 0.5680 & -62.1386 & 0.4500 & 17.4695 & 0.8120 \\
\hline
\end{tabular}

Fuente: Elaboración propia en base al SISREG y la Planilla Electrónica

La interpretación del modelo según los parámetros estimados y sus respectivas variables se detalla a continuación: 
- Hay una correlación positiva entre experiencia (plazos de los contratos) y el nivel de salario de la persona. Esto quiere decir que cuando una persona tiene mayor experiencia, ella registra mayores niveles de salario. Los beneficiarios que alcanza un año más de experiencia laboral (plazo de contratos) en promedio ganan S/ 81.50 más.

- Hay una correlación negativa entre sexo y nivel de salario de la persona. Esto quiere decir que cuando una persona es mujer su nivel de salario es menor. Las beneficiarias (mujeres) ganan en promedio S/ 110.25 menos de salario que los beneficiarios hombres.

- Hay una correlación positiva entre la edad y el nivel de salario de la persona. Esto quiere decir que, si una persona tiene más edad, también la persona tiene mayor salario. Cada año que suma a la edad en promedio equivale a S/ 6.86 más.

- Hay una correlación positiva entre educación y nivel de salario. Esto quiere decir que a medida que una persona tiene mayor cantidad de años alcanzados en educación, tiende a tener mayor nivel de salario. Los beneficiarios que pasan de un nivel académico a uno mayor ganan en promedio S/ 27.65 más.

- Hay una correlación positiva entre el resultado de evaluación y el nivel de salario. Ello significa que cuando un beneficiario ha logrado certificar sus competencias laborales, tiende a tener mayor nivel de salario. Los beneficiarios que lograron la certificación de competencias laborales ganan en promedio S/ 42 más que aquellos que no lograron salir competentes laboralmente.

- Hay una correlación negativa entre estado laboral y el nivel de salario. Ello implica que cuando la persona evaluada fue subempleada o empleada, ha ganado menores niveles de salario que aquellas que tuvieron la condición de desempleados. El cambio de estado laboral de empleado a desempleado implica un salario menor en un valor promedio de S/ 78.35

- Hay una correlación positiva entre el tipo de trabajador y el nivel de salario. Esto significa que una persona que es dependiente tiene 
mayor salario. Los beneficiarios que son dependientes ganan en promedio S/ 265 más que aquellos que son independientes.

- Hay una correlación negativa entre la inversión del Programa y el nivel de salario. Esto implica que las personas evaluadas con montos de inversión mayores, presentan menores niveles de salario. En promedio la diferencia es de S/ 4, la cual es pequeña.

- Hay una correlación negativa entre el ámbito geográfico y el nivel de salario. Esto implica que una persona que vive en un ámbito urbano gana en promedio S/ 133 más.

De los resultados obtenidos, es preciso considerar el modelo que toma en cuenta al salario como la variable dependiente dado que el primer modelo presenta dos variables no significativas en dos muestras.

Del segundo modelo se ha podido estimar un parámetro importante concerniente a la empleabilidad de los beneficiarios del servicio de Certificación de Competencias Laborales como es el impacto en el salario. Sin embargo, resulta relevante resaltar que este parámetro presenta un valor cercano con lo demostrado según el análisis de varianza de la sección anterior.

Tabla 8

Comparación de los indicadores estimados

\begin{tabular}{|l|l|l|}
\hline Variable & $\begin{array}{l}\text { Análisis de Varianza de } \\
\text { diferencia de Promedios }\end{array}$ & $\begin{array}{l}\text { Modelo de Regresión Lineal } \\
\text { (Estimador para el resultado de evaluación) }\end{array}$ \\
\hline $\begin{array}{l}\text { Diferencia en el nivel de } \\
\text { experiencia laboral }\end{array}$ & 3 meses y 22 días & $\begin{array}{l}\text { Muestra 1: 1 mes y 2 días (No significativo) } \\
\text { Muestra 2: 2 meses y 7 días } \\
\text { Muestra 3: 1 mes y 16 días } \\
\text { Muestra 4: 1 mes y 10 días (No significativo) }\end{array}$ \\
\hline $\begin{array}{l}\text { Diferencia en el nivel de } \\
\text { salario }\end{array}$ & S/ 70.30 & $\begin{array}{l}\text { Muestra 1: S/ 40.20 } \\
\text { Muestra 2: S/ 30.90 } \\
\text { Muestra 3: S/ 39.00 } \\
\text { Muestra 4: S/ 58.00 }\end{array}$ \\
\hline
\end{tabular}

Fuente: Elaboración propia en base al SISREG y la Planilla Electrónica

En la Tabla 8 se puede apreciar que para el caso del análisis de varianzas (ANOVA) se probó que, entre los grupos de competentes y no competentes, la diferencia en el nivel de experiencia laboral es de 3 meses y 22 días, mientras que para el caso de la diferencia de salarios es de S/ 70.30. 
En cuanto al modelo de regresión lineal estimado, para la variable "resultado final de evaluación", dichas diferencias son de 3 meses y S/ 64.46. Los resultados estimados con los modelos de regresión lineal presentan cierta proximidad a las diferencias comprobadas con el ANOVA, lo que nos sugiere que hay una buena especificación de los modelos econométricos. La brecha entre ambos cálculos son el resultado de las variables exógenas que se han tomado en cuenta para poder "filtrar" el parámetro de impacto del resultado de la certificación de competencias laborales.

En el caso de la variable dependiente de la experiencia laboral, la diferencia es de alrededor de 1.5 a 2 meses si tomamos válido los cálculos de las muestras 2 y 3 . Entonces, el impacto de la certificación "filtrada" está entre 1 y 2 meses más de diferencia entre las personas no competentes y competentes.

En el caso del modelo que toma en cuenta la variable salario, las diferencias son entre S/ 40 y S/ 10 por muestra, entonces el impacto de la certifica "filtrada" está entre S/ 30 y S/ 58. Esto dependiendo de las muestras que se tome en cuenta.

Con este resultado se prueba que la variable "resultado de evaluación final" tiene poder explicativo para la variabilidad del nivel de salario de las personas que han resultado competentes laboralmente en comparación a las que no resultaron competentes laboralmente, mostrando un impacto entre S/ 30 y S/ 58. Sin embargo, para el caso de la variable "experiencia laboral", el resultado de la certificación de competencias laborales no es claro pues presenta dos estimaciones no significativas en dos muestras.

\section{Conclusiones}

Desde las perspectivas teóricas planteadas acerca de la certificación de competencias laborales, así como de la visión planteada en el Modelo de Spencer, se pudo esbozar dos hipótesis de trabajo para el presente estudio del servicio de Certificación de Competencias Laborales del Programa Nacional para la Promoción de Oportunidades Laborales "Impulsa Perú".

La primera de ellas planteó que la certificación de competencias laborales tiene una relación con el tiempo de permanencia de una persona en el mercado laboral. A partir del estudio de los datos de los beneficiarios del 
servicio y la planilla electrónica (SUNAT), se pudo concluir que, de acuerdo a la Densidad de Kernel y el análisis de varianza, la permanencia en el mercado laboral (contratos registrados) ha tenido una diferenciación relativamente significativa entre las personas que resultaron competentes y aquellas que no lograron resultar competentes. Esta diferencia es en promedio 3 meses y 22 días más que aquellas que no resultaron competentes laboralmente. En el entorno de los grupos poblacionales que atiende el Programa, para una persona en condición de desempleo o subempleo la posibilidad de trabajar 3 meses y 22 días más resulta de vital importancia. Por otro lado, esta diferencia observada es efecto de múltiples factores, los cuales están correlacionados. Por ello, al plantear el modelo de regresión lineal con la variable dependiente "experiencia laboral", no se pudo comprobar que la variable "resultado de evaluación final" tenga poder explicativo dado que en dos de las muestras estimadas los parámetros calculados salieron no significativos. Sin embargo, tomando como válidos los resultados de las dos muestras significativas, la diferencia hipotética sería entre1 y 2 meses más a favor de las personas competentes.

La segunda hipótesis esbozada se fundamentó en el Modelo de Spencer, el cual expresa que la certificación es un mecanismo de señal para el mercado laboral a fin de que los empleadores sepan el nivel de productividad de un (posible)empleado. En definitiva, esto quiere decir que, si una persona logra demostrar sus habilidades y competencias laborales de manera certificada, entonces posee una herramienta que puede hacer notar alguno de los componentes de su empleabilidad a fin de mejorar el nivel de su salario. Usando el análisis de varianza se pudo constatar que el servicio de Certificación de Competencias Laborales ha tenido una relación significativa entre las personas que resultaron competentes y mayores niveles de salario (registrados en planilla electrónica) en promedio por S/ 70.3 más que aquellas que no resultaron competentes laboralmente. Nuevamente, en el entorno de los grupos poblacionales que atiende el Programa, para una persona en condición de desempleo o subempleo la posibilidad de mejorar sus ingresos en un valor promedio de S/ 70 más resulta de vital importancia. Sin embargo, considerando que dicha diferencia salarial es resultado de múltiples factores correlacionados, se planteó un modelo de regresión lineal, con el cual se pudo comprobar que la variable "resultado de evaluación final" tiene poder explicativo estimando un valor de diferencia entre S/ 30 y S/ 58 dependiendo de la muestra tomada en 
cuenta. La brecha entre la estimación del ANOVA y la regresión lineal es explicada por las demás variables planteadas en el modelo, como son la edad, el nivel educativo, el tipo de trabajo, entre otros.

A partir de estos resultados, es importante considerar que las personas con competencias laborales certificadas tienden a correlacionarse con un mejor resultado laboral, expresados a través de la permanencia en el mercado laboral (para el caso de dos muestras) y el mayor nivel de salario (para las cuatro muestras). Es por ello que, los responsables del servicio deben desarrollar estrategias conducentes a que se puedan certificar la mayor tasa de beneficiarios. Si bien se alcanza un $90 \%$, es importante que se continúe y se promueva el desarrollo de capacitaciones y reevaluación de las competencias laborales de aquellos que no aprobaron durante la primera etapa. Si en caso ello no fuera posible debido al costo que implica, se podría reforzar el equipo de focalización a fin de convocar a las personas que cumplen con los criterios específicos a fin de que tengan mayor éxito en la evaluación de sus competencias laborales. Además, desarrollar mecanismos normativos que puedan mejorar la ejecución de las actividades y el filtro de las personas a ser afiliadas al servicio.

Asimismo, los resultados mostrados resaltan la pertinencia del servicio de competencias laborales como aquel orientado a la mejora de la empleabilidad a través de la incorporación de una herramienta de señalización (signaling) de la cual puede disponer el beneficiario para afrontar con mejores resultados la dinámica del mercado laboral. En consecuencia, este mecanismo debe ir de la mano, con la capacitación laboral para poder tener un impacto sobre la productividad de la Población, es decir, la certificación promueve perfiles valorados por el mercado que marcan la ruta de los procesos de capacitación laboral.

Finalmente, se ha analizado el hecho que la certificación de competencias laborales contribuye como un mecanismo de vinculación entre la oferta y la demanda laboral en la medida en que logra mejorar los niveles de salario y experiencia laboral de aquellos que han adquirido dicho instrumento. Por ello, en base a la experiencia de países como Chile y México, y a los resultados del servicio brindado en el Ministerio de Trabajo y Promoción del Empleo a través del Programa Nacional para la Promoción de Oportunidades Laborales “Impulsa Perú”, es necesaria la creación, a nivel de Ley, 
de un sistema nacional de certificación de competencias laborales a fin de mejorar el desempeño en el mercado laboral y el mercado de capacitación y entrenamiento para el trabajo. Este mecanismo debe estar planteado en la próxima versión de la Política Nacional del Empleo y ser tomada como un tema prioritario en el Ministerio de Trabajo y Promoción del Empleo.

\section{Referencias}

Acemoglu, D. y Autor, D. (2015). Lectures in Labor Economics. Boston: Massachusetts Institute of Technology, 299p.

Álvarez Medina, L. y De la O Pérez, C. (2005). Evaluación y certificación de competencias laborales en México. México D.F.: Universidad Nacional Autónoma de México, 22p.

Berenson, M. y Levine, D. (1996). Estadística Básica en Administración. México D.F.: Pearson, 943p.

Bertrand, 0. (1997). Evaluación y certificación de competencias y cualificaciones profesionales.Buenos Aires: UNESCO, 127p.

Cahuc, P. y Zylberberg , A. (2001). Labor Economics.Boston: Massachusetts Institute of Technology, 1043p.

ChileValora. (2018). 8 años sembrando valor en Chile. Santiago de Chile: Comisión del Sistema Nacional de Certificación de Competencias Laborales, 100p.

Escobar Valencia, M. (2005). Las competencias laborales: La estrategia laboral para la competitividad de las organizaciones.Bogotá: Universidad del Valle, $25 \mathrm{p}$.

Forrier, A. y Sels, L. (2003). The concept of employability: a complex mosaic.Belgica: Human Resources Development and Management, Vol. 3, No 2, p. 102-124

Gómez Vélez, M. (2012). La noción de empleabilidad: una mirada desde la perspectiva de las organizaciones. Katharsis, Vol. 13, p. 63-83.

Gujarati, D. y Porter, D. (2010). Econometría. México D.F.: McGraw Hill, 946p.

Herman, C. (2015). Returning to STEM: gendered factors affecting employability for mature women students. Journal of Education and Work, Vol. 6,p. 571-591.

Mertens, L. (1996). Competencia Laboral: sistemas, surgimiento y modelos. Montevideo: Cinterfor - OIT, 133p.

O'Brien, E. y Silva, R. (2012). What is employability? Birmingham: University of Birmingham, 12p. 
Organización para la Cooperación y el Desarrollo Económico - OCDE (2008). Sistema de Cualificaciones. Puentes para el aprendizaje a lo largo de la vida. París: Instituto Nacional de las Cualificaciones, 230p.

Olivera Vidal, M.; Corvalán, M.; Ortega, T.; Loyola, A. y Illanca, A. (2017). Estudio sobre usos y resultados de la certificación de competencias en las empresas que participan en el Sistema Nacional de Certificación de Competencias. Santiago de Chile: ChileValora, 274p.

Ordaz Sanz, J.; Melgar Hiraldo, M. y Rubio Castaño, C. (2010). Métodos Estadísticos y Econométricos en la Empresa y para Finanzas. Sevilla, España: Universidad Pablo de Olavide, 267p.

Pitan, S. y Atiku, S. (2017). Structural determinants of student's employability: influence of career guidance. South African Journal of Education, Vol. 37, No 4,p. 1-13.

Rodríguez Cuba, J. (2009). Índice de empleabilidad de los jóvenes.Madrid: Fundación Carolina, 59p.

Ronald, W. (2004). Employanility, Local Policy Interpretations and The New Localism. Londres: McQuaid Employment Research Institute, Napier University, 8p.

Schleider, A. y Caddy, J. (2016). OECD Skills Strategy. Informe de Diagnóstico para Perú.Lima, Perú: OECD, 12p.

Spence, M. (1973). Job Market Signaling. The Quaterly Journal of Economics, Vol. 87, No 2, p. 335-374.

Tamayo Taype, M. Á. (2012). Importancia e impacto de la Certificación de Competencias en la sostenibilidad del crecimiento productivo y económico del país. Lima: Certification of System \& People, 60p.

Vargas, F. (2012). Competencias Laborales y Diálogo Social. Santiago de Chile: Cinterfor, 18p.

Wooldridge, J. (2006). Introducción a la Econometría: Un enfoque moderno. Madrid: S.A. Ediciones Paraninfo, 890p.

\section{Notas al final}

1 El motivo de este documento de investigación es analizar la importancia que tiene la certificación de competencias laborales como mecanismo de señalización en el mercado laboral, lo cual repercute en la mejora de la empleabilidad vía niveles de salario y mayores niveles de experiencia laboral para una persona. Este artículo corresponde al tipo reflexivo pues en él se analiza de manera analítica e interpretativa las acciones ejecutadas por el Programa Impulsa Perú del Ministerio de Trabajo y Promoción del Empleo durante los años 2011-2016.

2 Economista, egresado de la maestría de economía con mención en gestión y políticas públicas en la Universidad Nacional Mayor de San Marcos. Especialista en Planificación con cuatro años de experiencia laboral en el Ministerio 


\section{Christian OMar Silva Suárez}

de Trabajo y Promoción del Empleo en temas de planificación, monitoreo y evaluación de programas laborales. Tlf: 012650907. Correo: cislvas@trabajo.gob.pe

3 De acuerdo al MTPE corresponde a la estandarización del desempeño laboral de acuerdo a criterios de calidad establecidos por el sector productivo. Para ello se determina la demanda ocupacional, se elaboran mapas de macro procesos y procesos, mapas funcionales de sectores económicos y perfiles ocupacionales/cualificaciones en base a competencias laborales.

4 El autor lo obtuvo de la Confederación de Industria Británica (CBI), 1999, pág 1.

5 El autor lo obtuvo de Canadian Labour Force Development Board, 1994.

6 Premio Nobel de Economía en el año 2001 por sus trabajos sobre información asimétrica en los mercados.

7 Los índices son las características de los trabajadores que estos no pueden cambiar libremente como, por ejemplo, el sexo, la raza, la edad, etc. 